\title{
Grouped and Segmented Equalization Strategy of Serially Connected Battery Cells
}

\author{
Haolin $\mathrm{Li}^{1, \mathrm{a}}$, Guojing Dong ${ }^{2, \mathrm{~b}}$ and Qingliang Hong ${ }^{3, \mathrm{c}}$ \\ ${ }^{[1,2,3]}$ School of Electric Power Engineering, North China Electric Power University, \\ Baoding 071000, China;

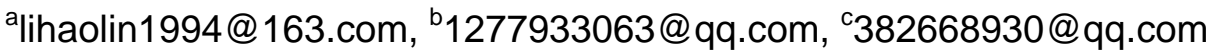

Keywords: grouped, segmented, balancing, local balance.

\begin{abstract}
The imbalance of serially connected battery cells can easily lead to overcharge and over discharge, which can not only shorten the life of the battery pack but also reduce the energy efficiency. In this paper, aimed at the merit and demerit of existing measures, a new balancing method based on grouping and segmentation is proposed. By grouping the battery pack, the relationship between the effect of equilibrium and the number of balancing devices can be balanced, and the balancing current can be reduced. And then, the segmented balance is used to avoid the local balance in the group. The method is simulated and verified in this paper. For six series of $6 \mathrm{~V}, 4.5 \mathrm{Ah}$ lead-acid batteries, this new measure can prolong discharge time of $24.7 \%$, and reduce $44.9 \%$ of the monomer balancing current compared with the measure of using only one set of equalization device.
\end{abstract}

\section{Introduction}

Practice showed that life of the battery made of multiple cells is greatly shorten, compared with individual cells, this is mainly because cells in the process of charging and discharging are not completely in synchronous. For multicellular battery, the minute differences between cells are likely to make the whole battery's performance decline. In these situations, weaker cells can be overcharged and over discharged, which causing them to become even weaker, until cause the collapse of the whole battery.

Overcharge and over discharge are not only a waste of energy, but a serious loss of battery life. In order to alleviate this phenomenon and improve the performance of a battery, cell balancing methods which can equalize the cell voltage of battery cells in a stack were born. At present, there are two main methods of charge balancing: passive and active balancing. The method that we put forward in this paper, based on active balancing, using DC-DC transform devices with smaller energy consumption. In addition, in order to overcome shortcomings of traditional charge balancing method, reduce the number of DC-DC converter and simplify the circuit, grouped and segmented equalization strategy is used.

\section{Existing balancing methods}

Active cell balancing deliver energy from the cells with higher voltage to lower cells, while passive balancing methods remove the excess energy from the higher cells through a resistant until the charge matches those of the lower cells in the pack ${ }^{[1,2]}$.

Passive cell balancing.In the process of battery charging, there will always be a variety of factors make battery cells' voltage not equal. Passive method is using resistance to consume higher voltage of some cells, until all the cells have equal voltage. When it detects a difference between cells and bypass resistor of the high voltage cell will be accessed. And through the shunt resistor, these cells' voltages grow slowly and ultimately achieve balance.

This method is simple and easy to control, but passive balancing is very slow and the excess energy during balancing is dissipated in heat. In addition, with passive balancing a battery stack can only be equalized by discharging the cells until all cell voltages equal the lowest one. 
Active cell balancing. Active cell balancing is based on the active transport of energy among cells. Energy can transfer between single cells as well as between cells and the battery stack. There are several types of active balancing methods based on the type of energy transfer. For example, capacitive balancing and inductive balancing structures. And capacitive balancing include charge shuttling and flying capacitor. Inductive balancing structures use DC/DC converters to transfer charge between the battery cells. A bidirectional DC/DC converter is used to transfer energy for every two adjacent battery cells, as shown in Fig. ${ }^{\left[{ }^{[1]}\right.}$. In active cell balancing, energy can be transferred from the strongest cell to the whole battery or other cells, and from the battery or other cells to the weakest cell, thus, a weak cell can be supported by the other cells during discharging.

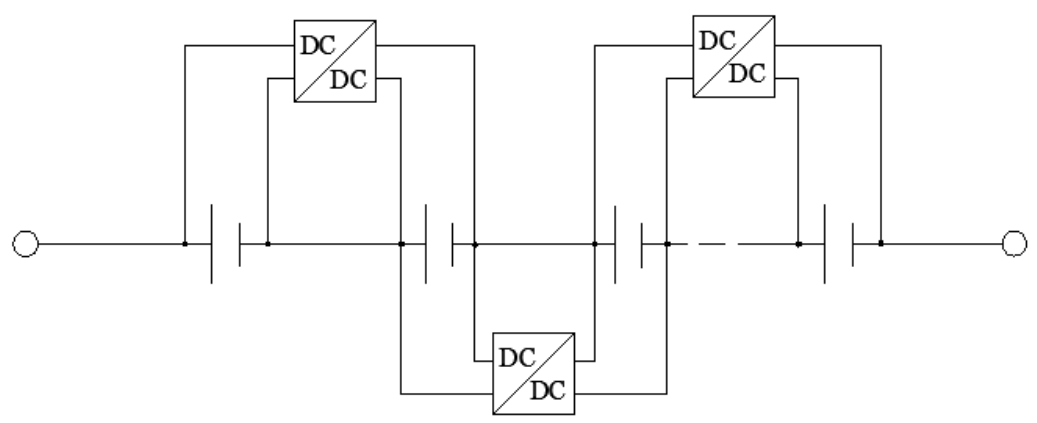

Fig.1 Active balancing with one DC/DC converter for every two adjacent battery cells

A significant disadvantage of the structure is if the strongest and the weakest cell are far away from each other, the energy has to be transferred through several DC/DC converters. While every cell with a DC/DC converter is more flexible and powerful, the number of DC/DC converters maybe too much and become a waste.

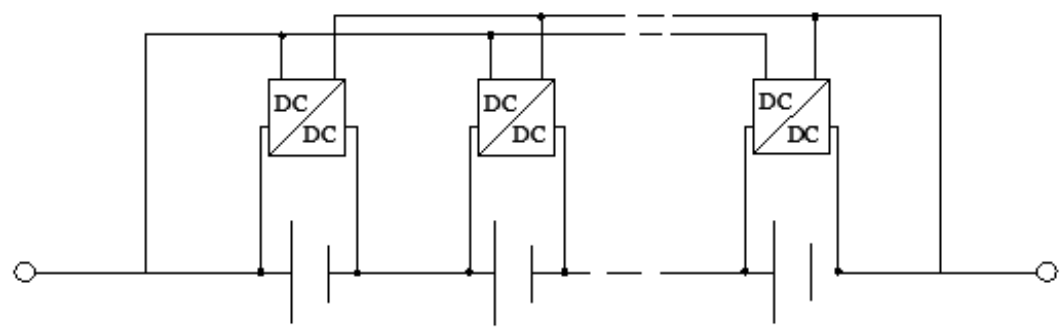

Fig.2 Active balancing strategy with DC/DC converters for every battery cell

Other two effective structures are shown in Fig.2 and Fig. $3^{[1,2,3]}$. Fig.2 have a balance device for each cell, which is flexible and powerful enough but too expensive and complicated. Fig. 3 is a simplification for Fig.2. It manages the battery pack with only one balance device. By transferring the energy from the whole battery stack to battery monomer, the group will tend to balance. But when the unbalance degree is high or the number of cell is large, this structure may be helpless.

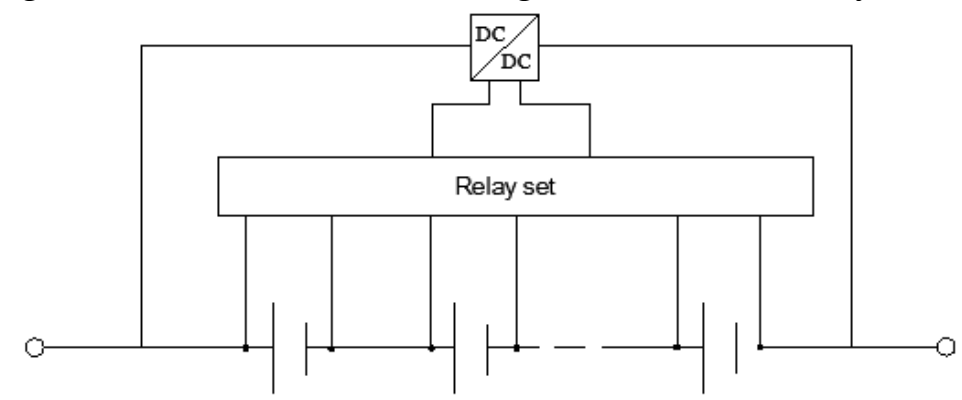

Fig.3 Active balancing strategy with only one DC/DC converters for all battery cells

\section{Grouped and Segmented Equalization Strategy}

Based on the advantages and disadvantages of the above schemes, we design a new method called grouped and segmented equalization strategy shown in Fig.4. The Battery pack is divided into some groups, and each group is configured with a balancing device. The circuit adopts a simplified switch 
structure ${ }^{[4]}$. The balancing device transfers the energy from the whole battery stack to battery monomer which has the lowest state of charge (SOC).

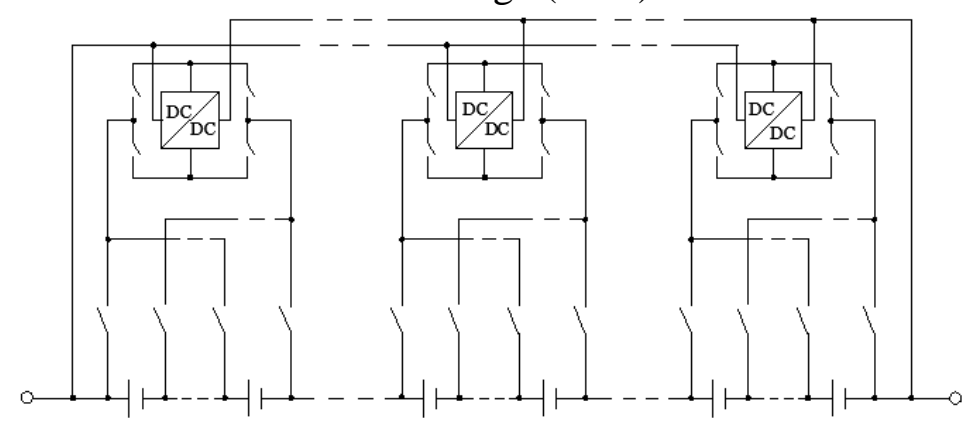

Fig.4 Grouped and segmented equalization strategy

By grouping the battery pack, the number of equalization devices is reduced in the case of equalization performance, and reduces the scope and burden of the device compared with Fig.3. On the basis of grouping, in order to avoid falling into local balance, we adopt the segmented balance strategy.

Assuming that the battery pack is divided into $N$ groups, and every group has $M$ cells.

Early stages.In the early stage of equalization, the degree of imbalance is relatively large. Using the grouped equalization strategy, the energy is transferred to the cells with the smallest SOC among the $M$ cells within the group. Local balance is achieved at this stage, and there are $N$ cells accept the energy supplement during the same period of time.

Late stage. When the SOC range of the group is small enough, the group can be considered to have reached a balance, then the system turn to global equalization. At this stage, if the group $i(i \in 1,2, \ldots, N)$ No. $j(j \in 1,2, \ldots, M)$ battery is the one which has the lowest SOC in the whole battery pack, the energy will be transferred to it. Global balance is achieved at this stage, and there are only one cell accept the energy supplement during the same period of time.

Simulation.To verify the feasibility of the method, we take the discharge process as an example, and use Matlab/Simulink to build a simulation model shown in Fig. 4. The simulation model has six series of $6 \mathrm{~V}, 4.5 \mathrm{Ah}$ lead-acid battery cells, and it is divided into two groups. Each group is equipped with a balanced module(the light blue module ), which driven by center control module(the light yellow module). By setting different initial capacity of the battery pack, the unbalance of the battery can be simulated, and the balance current can be controlled by the green module. When the green module gain is set to 0 , that is to say, model exit the equilibrium mode. In this simulation, the initial SOC range is $12 \%$, and the ratio of the balancing current to the bus current (gain) is 0.27 .

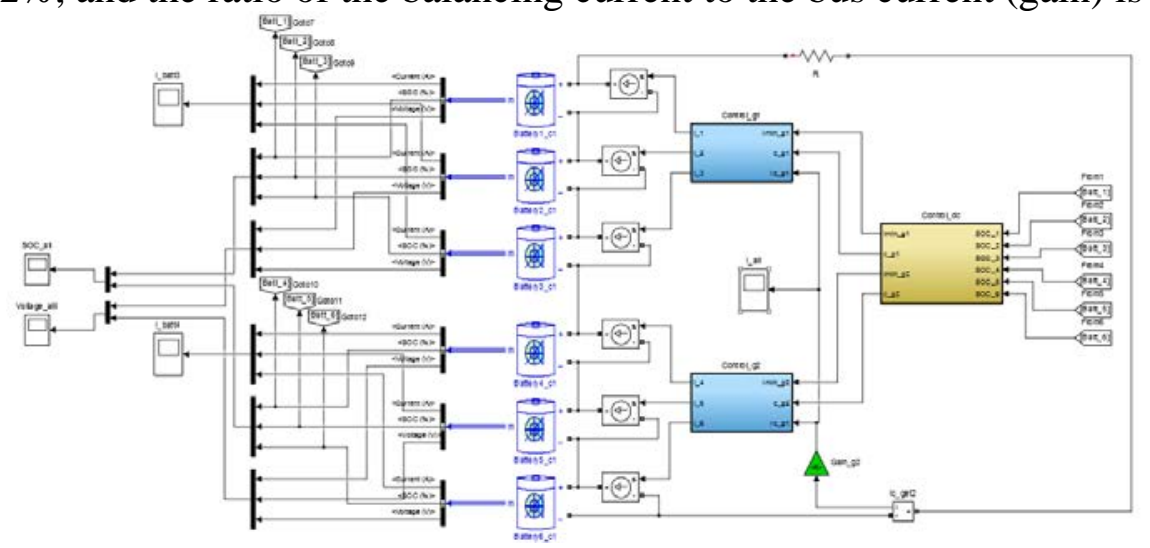

Fig.5 Simulation model for grouped and segmented equalization strategy

Local balance phenomenon. When the balance of the discharge process is carried out only by using the packet equalization, balance modules of every group provide energy to the battery with the smallest SOC within the group, which makes the SOC of cells in the group gradually tend to be consistent. In this case, cells in the same group reach the completion of the discharge at nearly the same time. However, within the group reached a balance, but the whole battery pack gradually separate by group, failed to achieve a global balance. 


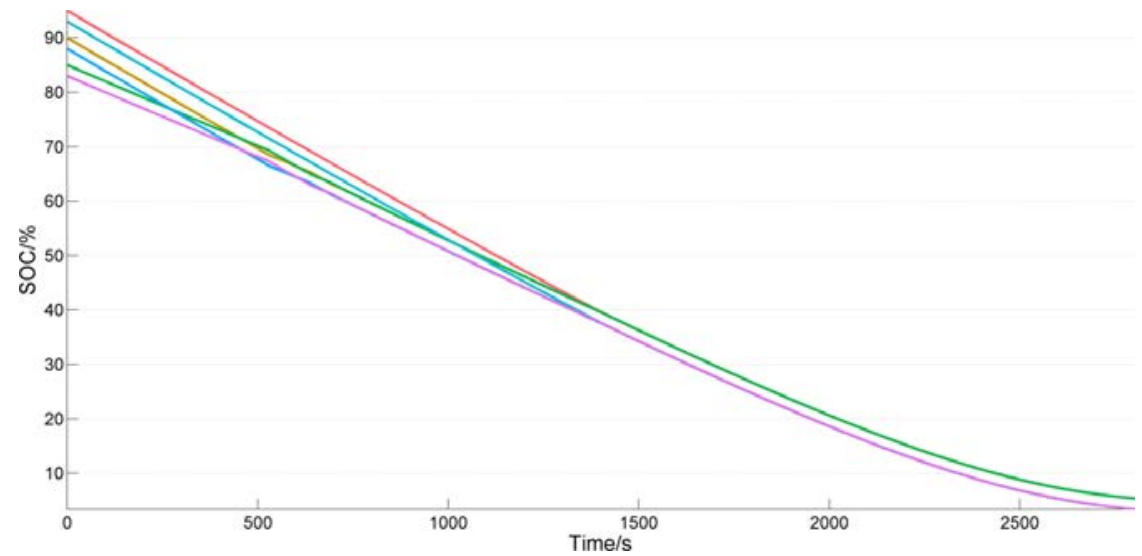

Fig.6 Local balance phenomenon for grouped equalization

Fig.7 shows the number of times that cells in the second packet are balanced. Compared with Fig.6, we can find that the difference of the SOC of each cell in the group becomes smaller gradually. Especially after the 1500s, even the fourth cell which has the maximum SOC is also involved in the balance, which means the group has almost reached a balance. Then cells in the same group catch one another in the discharged process and cannot break out from loops, so the local balance happened.

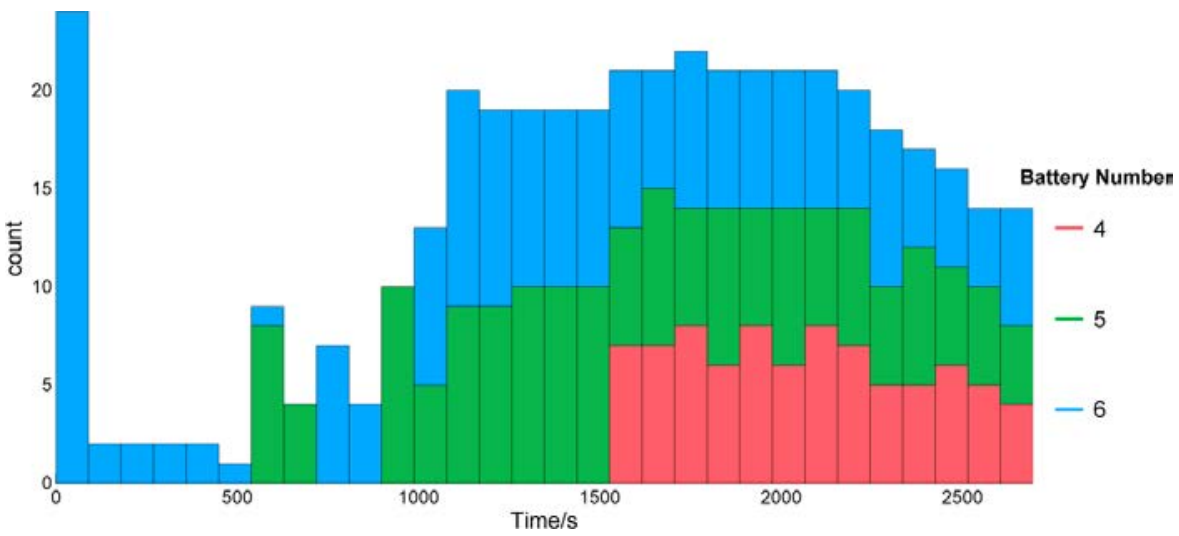

Fig.7 Stacked histogram of the number of times that cells in the second packet are balanced

In order to make up for the local balance phenomenon, the segmented balance strategy is proposed. By reuniting each group to the structure shown in Fig.4 by program control, the gap between the groups can be smaller. And in this stage, the balance degree has been significantly improved, so the system can balance the battery pack with a smaller current.

SOC and voltage changes after adopting the balance strategy are shown in Fig.8.

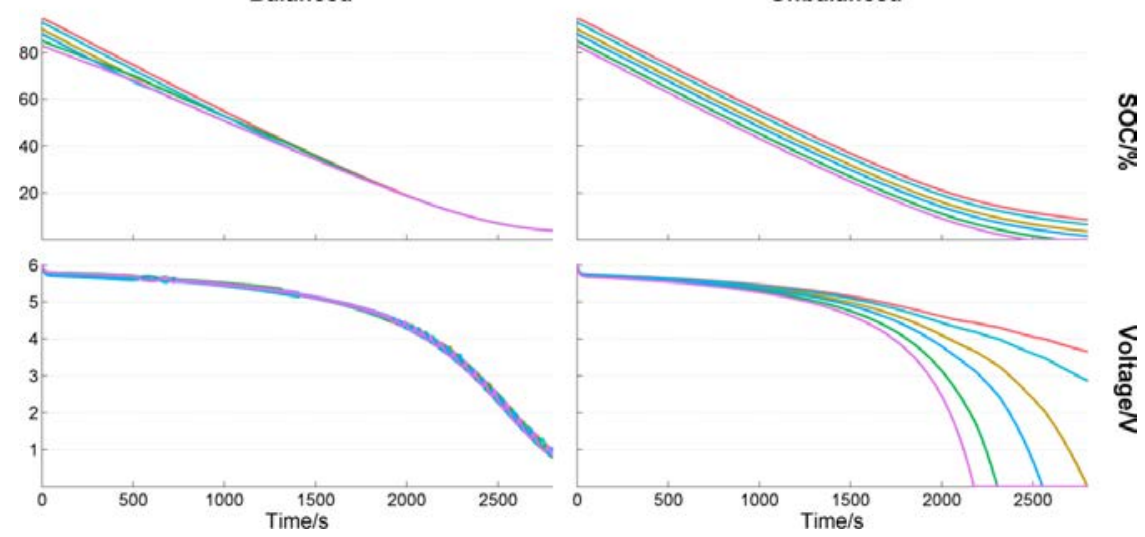

Fig.8 SOC and voltage changes after adopting grouped and segmented equalization strategy

Assuming the discharge cut-off voltage is $5 \mathrm{~V}$, the structure in Fig.3 and grouped and segmented equalization strategy are simulated. The former's balancing current accounts for 0.49 , and the discharge time was prolonged by $24.094 \%$ compared with using no balancing measure. The latter's balancing current accounts for 0.27 , and the discharge time was prolonged by $24.7 \%$. Hence, grouped 
and segmented equalization strategy can achieve a remarkable balancing results with lower balancing current.

\section{Summary}

In the current balancing strategy, the active balance strategy can balance the serially connected battery cells more effective. Based on the strengths and weaknesses of the main active balance strategy, this paper proposes the grouped and segmented equalization strategy, and the feasibility of the strategy is verified by simulation. When the initial SOC range is $12 \%$, compared with using only one balancing device, the prolongation of the discharge time increased by $0.6 \%$, and the proportion of balancing current is reduced by $44.9 \%$. This means that the work load and power consumption of the device can be significantly reduced.

\section{Acknowledgments}

This work was financially supported by "Undergraduate Training Program for Innovation and Entrepreneurship(20152009)”.

\section{References}

[1] M Einhorn, et, al. Charge balancing of serially connected lithium-ion battery cells in electric vehicles[J]. Elektrotechnik \& Informationstechnik, 2012, 129(3): 167-173

[2] S.S. Williamson. Energy Management Strategies for Electric and Plug-in Hybrid Electric Vehicles[M]. New York: Springer-Verlag, 2013.

[3] Yuan X., Huang W, He Y.P. and Huang P. Charge and discharge equalization method for lead acid battery[J]. Journal of Changsha Communication College, 2005, 21(3): 11-15

[4] Wang W. Research on software and hardware of lead acid battery management system[D]. Hangzhou:Zhejiang University, 2005.

[5] He Y.P. Research on balance control of charge and discharge in electric vehicle power battery[D]. Changsha:Changsha University of Science and Technology, 2005.

[6] Huang W. Experimental study on balance control of series discharge in lead acid battery[D]. Changsha:Changsha University of Science and Technology, 2005.

[7] CUI J.Y. and ZHANG X. W. Two-Layer Distributed Equalization Management System For Electric Vehicle Power Battery[J]. International Journal of Automotive Technology, 2015, 16(6): 1007-1016

[8] Wu H.B, Gu X., Zhao B., Zhang X. S. Whole process simulation of battery charge and discharge management[J]. Journal of electronic measurement and instrument, 2014, 28(8): 843-849 